\title{
Robust Model Predictive Control of Networked Control Systems under Input Constraints and Packet Dropouts
}

\author{
Deyin Yao, ${ }^{1}$ Hamid Reza Karimi, ${ }^{2}$ Yiyong Sun, ${ }^{3}$ and Qing Lu ${ }^{1}$ \\ ${ }^{1}$ College of Engineering, Bohai University, Jinzhou, Liaoning 121013, China \\ ${ }^{2}$ Department of Engineering, Faculty of Engineering and Science, University of Agder, 4898 Grimstad, Norway \\ ${ }^{3}$ Research Institute of Intelligent Control and Systems, Harbin Institute of Technology, Harbin, Heilongjiang 150080, China
}

Correspondence should be addressed to Deyin Yao; deyinyao2013@gmail.com

Received 22 May 2014; Accepted 4 June 2014; Published 16 July 2014

Academic Editor: Josep M. Rossell

Copyright (C) 2014 Deyin Yao et al. This is an open access article distributed under the Creative Commons Attribution License, which permits unrestricted use, distribution, and reproduction in any medium, provided the original work is properly cited.

\begin{abstract}
This paper deals with the problem of robust model predictive control (RMPC) for a class of linear time-varying systems with constraints and data losses. We take the polytopic uncertainties into account to describe the uncertain systems. First, we design a robust state observer by using the linear matrix inequality (LMI) constraints so that the original system state can be tracked. Second, the MPC gain is calculated by minimizing the upper bound of infinite horizon robust performance objective in terms of linear matrix inequality conditions. The method of robust MPC and state observer design is illustrated by a numerical example.
\end{abstract}

\section{Introduction}

Model predictive control (MPC) $[1,2]$ is an important method to handle control problems with systems having input, state, and output constraints [3-7]. Its current control action is obtained by solving an online, at each time instant, open-loop constrained infinite horizon optimization problem. The current state of the system is treated as the initial state of optimal control problems [8], and only the first optimal control sequence is implemented [9]. In this line of the research, a model is used to predict the future behavior of the real system and obtain an optimal control sequence which satisfies the input, state, and output constraints. So the model quality is very vital in the robust MPC.

From such a viewpoint, it is a significant problem to develop the MPC algorithms, which are robust against model uncertainties, and guarantee a certain control performance objective [10-17]. The type of MPC has been studied for many years [18-21]. Recently, MPC is an efficient method in automatic control theory and industrial processes. In [22], a robust MPC is presented for a class of uncertain systems and then applied to angular positioning system. Besides, the robust MPC developed based on explicit model uncertainty descriptions has been proposed [23]. As one of such descriptions, polytopic system model is considered to be an effective one for the uncertainty modeling of linear timevarying (LTV) systems.

With the increasing requirement of reliability, environmental sustainability, and profitability, we begin to apply the communication networks to the practical industrial process [24-31]. However, in the real systems, the measurement data may be transferred through multiple sensors. The sensors are connected to the controller via a network, which is shared by other networked control systems. Due to sensor aging and sensor temporal failure, the measured data may be transferred through many sensors in the real process via networks so that successive packet dropouts are unavoidable in the real industry process [32-34]. Moreover, another significant factor, input constraints, which stems from the restrictions of engineering equipment, cannot be ignored in industrial processes. Therefore, the robust MPC controller should be developed by considering packet dropouts and constraints.

In this paper, we describe an industrial process system as the linear time-varying system with packet dropouts from the MPC controller and dynamic output controller to the plant. Furthermore, a Bernoulli random binary distribution with known probabilities is used to describe the packet dropouts 
phenomena. Thus, the MPC controller can be analyzed and designed to guarantee that the closed-loop system is stochastically stable. Additionally, the Lyapunov function is adopted to handle the problem of designing the controller and state observer, which make the result less conservative. A numerical example is proposed to prove the effectiveness of the design method. The main merit of this paper is the following one: a robust MPC controller is developed for a control system with uncertainties, saturations, and packet dropouts under time-varying probabilities.

The organization of the paper is given as follows. The considered problem is denoted in Section 2. Section 3 formulates the infinite horizon robust performance objective. The procedure of obtaining the state observer gains is represented in Section 4. And the MPC controller gain and dynamic output controller are represented in Sections 5 and 6 , respectively. We employ two numerical examples to certify the feasibility of the mentioned method in Section 7. Finally, Section 8 presents the conclusion.

Notation. The notation used in the paper is standard. The superscript " $T$ " represents the matrix transposition and $\mathbb{R}^{n_{x}}$ shows the $n_{x}$-dimensional Euclidean space. And $I$ and 0 denote the identity matrix and zero matrix, respectively. The notations $P>0$ and $Q>0$ mean that $P$ and $Q$ are real symmetric and positive definite; the notation $\|A\|$ refers to the norm of matrix $A$ that is defined by $\|A\|=\sqrt{\operatorname{tr}\left(A^{T} A\right)}$, where "tr" stands for the trace operator. $\|\cdot\|_{2}$ refers to the usual Euclidean vector norm. The symbol "*" denotes the elements below the main diagonal of a symmetric block matrix. Prob $\{\cdot\}$ means the occurrence probability of the event ".. $E\{x\}$ and $E\{x \mid y\}$ represent the expectation of event $x$ and the expectation of $x$ conditional on $y$, respectively.

\section{Problem Formulation}

First, we consider the linear time-varying system as follows:

$$
\begin{gathered}
x(k+1)=A(k) x(k)+B(k) u(k), \\
y(k)=C x(k), \\
{[A(k) \quad B(k)] \in \Omega,}
\end{gathered}
$$

where $x(k) \in \mathbb{R}^{n_{x}}$ is the plant state vector; $y(k) \in \mathbb{R}^{n_{y}}$ is the output of the plant; $u(k) \in \mathbb{R}^{n_{u}}$ is the control input; $A(k)$, $B(k)$, and $C$ are system parameters. $\Omega$ is a given set. For the polytopic systems, the set $\Omega$ is the polytope:

$$
\Omega=\operatorname{Co}\left\{\left[\begin{array}{ll}
A_{1} & B_{1}
\end{array}\right],\left[\begin{array}{ll}
A_{2} & B_{2}
\end{array}\right], \ldots,\left[\begin{array}{ll}
A_{L} & B_{L}
\end{array}\right]\right\},
$$

where Co represents the convex hull. Besides, if $[A(k) B(k)] \in \Omega$, then for $\lambda_{i} \geq 0, i=1,2, \ldots, L$, and $\sum_{i=1}^{L} \lambda_{i}=1,[A(k) B(k)]=\sum_{i=1}^{L} \lambda_{i}\left[A_{i}(k) B_{i}(k)\right]$. When $L=1$, it means that the system in (2) is becoming a linear time-invariant system.

In this paper, the system matrices $[A(k) B(k)]$ are known clearly. However, the future matrices $[A(k+i) B(k+i)]$, $i \geq 1$, are indefinite but well known which change in a given polytope set $\Omega$. We assume that the linear discrete-time system (1) has input constraints [35], which satisfies at each instant $k \geq 0$, as follows:

$$
\|u(k+i)\|_{2} \leq u_{\max }, \quad k, i \geq 0,
$$

where $u_{\max }$ refers to the peak bound of the input $u$.

In this paper, we consider a state observer to estimate the state of the plant (1) as follows:

$$
\begin{aligned}
\widehat{x}(k+1) & =A(k) \hat{x}(k)+L[y(k)-C \widehat{x}(k)]+B(k) u(k), \\
\widehat{y}(k) & =C \widehat{x}(k),
\end{aligned}
$$

where $\widehat{x}(k) \in \mathbb{R}^{n_{x}}$ refers to the estimated state of $x(k)$ and $L$ refers to the state observer gain. It is assumed that the initial estimate state $\widehat{x}_{0}=\widehat{x}(0)$ and the initial output $y_{0}=y(0)$ are known exactly. Note that system matrices $\left[\begin{array}{ll}A(k) & B(k)\end{array}\right]$, output $y(k)$, and the estimated state $\widehat{x}(k)$, at each instant $k$, are well known so that $\widehat{x}(k+1)$ can be calculated by using (4). However, the estimated state $\widehat{x}(k+i+1), i \geq 1$, is uncertain.

The MPC controller is to be determined as follows:

$$
u_{c}(k+i \mid k)=K(k) \hat{x}(k+i \mid k), \quad i \geq 0,
$$

where $K(k)$ is the feedback control gain at time instant $k$. In MPC, only the first calculated control input $u_{c}(k \mid k)=$ $K \widehat{x}(k \mid k)$ is complemented. At the next sampling time $k+1$, the state $\widehat{x}(k+1)$ is measured and $K$ is repeatedly computed by the optimization.

The form of dynamic output controller is as follows:

$$
\begin{aligned}
\widehat{x}_{c}(k+1) & =A_{c} \widehat{x}_{c}(k)+B_{c} y_{c}(k), \\
u_{c}(k) & =C_{c} \widehat{x}_{c}(k),
\end{aligned}
$$

where $A_{c}, B_{c}$, and $C_{c}$ are the unknown parameters of dynamic output controller.

Combining (1), (6), and (10), the augmented system is given as follows:

$$
\begin{gathered}
\theta(k+1)=[\bar{A}(k)+\widehat{A}(k)] \theta(k), \\
y(k)=\bar{C} \theta(k),
\end{gathered}
$$

where $\theta(k)=\left[\begin{array}{ll}x^{T}(k) & \hat{x}^{T}(k)\end{array}\right]^{T}, \bar{C}=\left[\begin{array}{ll}C & 0\end{array}\right]$, and

$$
\begin{aligned}
& \bar{A}(k)=\left[\begin{array}{cc}
A(k) & \bar{\beta} B(k) C_{c} \\
\bar{\alpha} B_{c} C & A_{c}
\end{array}\right], \\
& \widehat{A}(k)=\left[\begin{array}{cc}
0 & \widehat{\beta}(k) B(k) C_{c} \\
\widehat{\alpha}(k) B_{c} C & 0
\end{array}\right] .
\end{aligned}
$$

In practical industrial processes, we have to not only consider the uncertainties in (2), but also consider a more important factor that is data loss. Data losses are inevitable in communication networks, because the measurement data may be transferred through many sensors in the real process.

Here, we consider a stochastic variable $\delta(k)$ to describe successive packet dropouts in a random way. The characteristic of the successive packet dropouts is the latest received 
data that will be sent to the state observer. If the data is not updated, the control input will keep unchanged. More important is that taking the packet dropouts into account is consistent with the real situation design in industry. Therefore, signals transmitted through the communication network to the estimated state observer can be expressed as

$$
u(k)=\delta(k) u_{c}(k),
$$

where $u_{c}(k)$ is the control input that is computed by using the feedback gain $K(k)$ which is determined at each instant $k . u(k)$ is the actual measurement signal of $u_{c}(k)$ and transmitted to the plant (1) and state observer (4). We assume that the stochastic variable $\delta(k)$ satisfies $\operatorname{Prob}\{\delta(k)=1\}=$ $E\{\delta(k)\}=\bar{\delta}$ and $\operatorname{Prob}\{\delta(k)=0\}=1-\bar{\delta}$. Let $\widetilde{\delta}(k)=\delta(k)-\bar{\delta}$. Then, $E\{\widetilde{\delta}(k)\}=0$ and $E\left\{\widetilde{\delta}^{2}(k)\right\}=\bar{\delta}(1-\bar{\delta}) \triangleq \phi^{2}$.

In a similar situation, we consider stochastic variables $\alpha(k)$ and $\beta(k)$ to describe successive packet dropouts in a random way:

$$
\begin{aligned}
& u(k)=\beta(k) u_{c}(k), \\
& y_{c}(k)=\alpha(k) y(k),
\end{aligned}
$$

where $u_{c}(k)$ is the control input, $u(k)$ is the actual measurement signal of $u_{c}(k)$ which is transmitted to the plant (1), and $y_{c}(k)$ is the actual signal of $y(k)$ which is transmitted to the dynamic output controller, respectively. We assume that the stochastic variables $\alpha(k)$ and $\beta(k)$ satisfy $\operatorname{Prob}\{\alpha(k)=1\}=$ $E\{\alpha(k)\}=\bar{\alpha}$ and $\operatorname{Prob}\{\alpha(k)=0\}=1-\bar{\alpha}$ and $\operatorname{Prob}\{\beta(k)=$ $1\}=E\{\beta(k)\}=\bar{\beta}$ and $\operatorname{Prob}\{\beta(k)=0\}=1-\bar{\beta}$, respectively. Let $\widehat{\alpha}(k)=\alpha(k)-\bar{\alpha}$ and $\widehat{\beta}(k)=\beta(k)-\bar{\beta}$, respectively. Then, $E\{\widehat{\alpha}(k)\}=E\{\widehat{\beta}(k)\}=0, E\left\{\widehat{\alpha}^{2}(k)\right\}=\bar{\alpha}(1-\bar{\alpha}) \triangleq l^{2}$, and $E\left\{\widehat{\beta}^{2}(k)\right\}=\bar{\beta}(1-\bar{\beta}) \triangleq m^{2}$, respectively.

The following Lemma is used in the process of proof.

Lemma 1. Let $F$ and $G$ and $H$ and $E$ be the real matrices of appropriate dimensions; then $F+G H E+E^{T} H^{T} G^{T}<0$ if and only if there exists a positive scalar $\varepsilon>0$ such that $F+\varepsilon^{-1} G G^{T}+$ $\varepsilon E^{T} E<0$ or, equivalently,

$$
\left[\begin{array}{ccc}
F & * & * \\
G^{T} & -\varepsilon I & 0 \\
\varepsilon E & 0 & -\varepsilon I
\end{array}\right]<0 .
$$

\section{Infinite Horizon Robust Performance Objective Analysis}

In this paper, the aim of the robust MPC is to determine the model predictive control law $\{u(k \mid k), u(k+1 \mid k), \ldots, u(k+$ $i \mid k)\}, i \geq 1$, which regulates the initial state of system reaching the origin, making the robust performance objective minimum in the worst case and ensuring that the closedloop system is asymptotically stable. In order to determine the control input $u(k)$ under input constraints, we minimize the performance objective $J_{\infty}(k)$ through minimizing its upper bound. Consider the performance objective by minimizing the infinite horizon objective function $J_{\infty}(k)$ as follows:

$$
\min _{u(k+i \mid k)} \max _{[A(k+i \mid k)} J_{B(k+i \mid k)] \in \Omega} J_{\infty}(k),
$$

where

$$
\begin{array}{r}
J_{\infty}(k)=E\left\{\sum _ { i = 0 } ^ { \infty } \left[y^{T}(k+i \mid k) R_{1} y(k+i \mid k)\right.\right. \\
\left.\left.+u^{T}(k+i k) R_{2} u(k+i k)\right]\right\},
\end{array}
$$

in which $R_{1}$ and $R_{2}$ are the positive-definite matrices that should be given firstly. It is worth noting that the choices of $R_{1}$ and $R_{2}$ are unclear, which are usually adjusted according to the practical situation.

We consider transferring the minimization of $J_{\infty}(k)$ into minimizing its upper bound. Therefore, we assume that the following inequality holds for all $y(k \mid k), u(k \mid k), i \geq 0$, satisfying the input constraints (3), and for any $[A(k+i) \quad B(k+$ i)] $\in \Omega, i \geq 0$ :

$$
\begin{aligned}
& E\{V(\widehat{x}(k+i+1 \mid k)) \mid \widehat{x}(k+i \mid k)\} \\
& -V(\widehat{x}(k+i \mid k)) \\
& \leq-E\left\{y^{T}(k+i \mid k) R_{1} y(k+i \mid k)\right. \\
& \left.\quad+u^{T}(k+i \mid k) R_{2} u(k+i \mid k)\right\},
\end{aligned}
$$

where $V(\widehat{x}(k))=\widehat{x}^{T}(k) P \widehat{x}(k)$ and $P>0$ is a symmetric matrix.

Proof. Firstly, we construct a Lyapunov function $V(\widehat{x}(k))=$ $\widehat{x}^{T}(k) P \widehat{x}(k)$, where $P>0$ is a symmetric matrix. We assume that $E\{V(\widehat{x}(\infty \mid k))\}=0$. By summing (14) from $i=0$ to $i=\infty$, we can obtain the following inequality:

$$
\begin{aligned}
& \max _{[A(k+i)} \max _{B(k+i)] \in \Omega, i \geq 0} J_{\infty}(k) \leq V(\widehat{x}(k \mid k)) \\
& -E\{V(\widehat{x}(\infty \mid k))\} \leq \widehat{x}^{T}(k \mid k) P \hat{x}(k \mid k) .
\end{aligned}
$$

Let $\gamma$ be the upper bound of $J_{\infty}(k)$ and we have the following inequality:

$$
\widehat{x}^{T}(k \mid k) P \widehat{x}(k \mid k) \leq \gamma
$$

In a similar situation, we consider transferring the minimization of $J_{\infty}(k)$ into minimizing its upper bound. Therefore, we assume that the following inequality holds for all $y(k \mid k)$, $u(k \mid k), i \geq 0$, satisfying the input constraints (3), and for any $[A(k+i) B(k+i)] \in \Omega, i \geq 0$ :

$$
\begin{aligned}
E\{V(\theta(k+i+1 \mid k)) \mid \theta(k+i \mid k)\} \\
-V(\theta(k+i \mid k)) \\
\leq-E\left\{y^{T}(k+i \mid k) R_{1} y(k+i \mid k)\right. \\
\left.\quad+u^{T}(k+i \mid k) R_{2} u(k+i \mid k)\right\} .
\end{aligned}
$$




\section{State Observer Design}

In this section, we consider the design of state observer. The form of state observer is (4). Therefore, the state estimation error is $e(k)=x(k)-\widehat{x}(k)$. Combining (1) with (4), we can derive the error dynamics as follows:

$$
e(k+1)=(A-L C) e(k) .
$$

Next we use LMI to determine the state observer gain $L$. First, we should construct a Lyapunov function $\widetilde{V}(e(k))$ as follows:

$$
\widetilde{V}(e(k))=e^{T}(k) \widetilde{P} e(k), \quad \widetilde{P}>0 .
$$

We assume that the Lyapunov function $\widetilde{V}(e(k))$ satisfies the following inequality:

$$
\widetilde{V}(e(k+1))-\rho^{2} \widetilde{V}(e(k))<-e^{T}(k) \widetilde{L} e(k),
$$

where $\rho(0<\rho<1)$ is a decay rate and $\widetilde{L} \geq 0$ is a weighting matrix which are determined by the designer. According to the assumption (20), the state observer gain $L$ can be obtained by the following theorem.

Theorem 2. If there exist $\widetilde{P}>0$ and $\widetilde{Q}=\widetilde{P} L$, one can derive the following inequality:

$$
\left[\begin{array}{cc}
\rho^{2} \widetilde{P}-\widetilde{L} & * \\
\widetilde{P} A-\widetilde{Q} C & \widetilde{P}
\end{array}\right]>0,
$$

where the state observer gain is derived by $L=\widetilde{P}^{-1} \widetilde{Q}$. Therefore, the estimated state $\widehat{x}$ can track the original system states $x$ and $\hat{x}(k) \rightarrow x(k)$ when $k \rightarrow \infty$.

Proof. Inequality (20) is equivalent to

$$
\rho^{2} \widetilde{P}-\widetilde{L}-\Pi^{T}(k) \widetilde{P} \Pi(k)>0
$$

where $\Pi(k)=A(k)-L C$. However, inequality (22) can be converted into the following form:

$$
\rho^{2} \widetilde{P}-\widetilde{L}-(\widetilde{P} \Pi(k))^{T} \widetilde{P}^{-1}(\widetilde{P} \Pi(k))>0 .
$$

By using Schur complement, we can obtain the following LMI constraint:

$$
\left[\begin{array}{ll}
\rho^{2} \widetilde{P}-\widetilde{L} & * \\
\widetilde{P} \Pi(k) & \widetilde{P}
\end{array}\right]>0
$$

Let $\widetilde{Q}=\widetilde{P} L$; inequality (24) is equivalent to

$$
\left[\begin{array}{cc}
\rho^{2} \widetilde{P}-\widetilde{L} & * \\
\widetilde{P} A(k)-\widetilde{Q} C & \widetilde{P}
\end{array}\right]>0 .
$$

It is established for all $[A(k) B(k)] \in \Omega$. And the state observer gain $L$ is obtained by $L=\widetilde{P}^{-1} \widetilde{Q}$.

\section{MPC Controller Design}

Now our goal is to design a robust MPC to generate an optimal control law so that the performance objective can be achieved. In the following, Theorem 3 is given to guarantee that the desired performance objective can be implemented by computing $K(k)$ in (5) for linear discrete-time systems (1) under constraints (3) and packet dropouts (9). We can obtain the following theorem.

Theorem 3. Assume that the uncertainty $\Omega$ is a prescribed polytope and the probabilities $\bar{\delta}$ take values from 0 to 1 . The control input (5) under a series of constrained input (3) that minimizes $J_{\infty}(k)$ is obtained by

$$
K=Y Q^{-1} \text {, }
$$

where $Q>0$ is symmetric and the upper bound $\gamma$ of $J_{\infty}(k)$ can be solved by the following minimization problem:

$$
\min _{u(k \mid k), Q, Y} \gamma
$$

subject to

$$
\begin{aligned}
& {\left[\begin{array}{cc}
1 & \widehat{x}^{T}(k \mid k) \\
* & Q
\end{array}\right] \geq 0,} \\
& {\left[\begin{array}{cccccccc}
-Q & 0 & \Gamma & Y^{T} B^{T} & Y^{T} R_{2}^{1 / 2} & Q^{T} C^{T} R_{1}^{1 / 2} & Q^{T} C^{T} & 0 \\
* & -\Lambda & 0 & 0 & 0 & 0 & Q^{T} C^{T} & 0 \\
* & * & -Q & 0 & 0 & 0 & 0 & \bar{L} \\
* & * & * & \frac{-Q}{\phi^{2}} & 0 & 0 & 0 & 0 \\
* & * & * & * & \frac{-\gamma I}{\bar{\delta}^{2}+\phi^{2}} & 0 & 0 & 0 \\
* & * & * & * & * & -\gamma I & 0 & 0 \\
* & * & * & * & * & * & -\varepsilon I & 0 \\
* & * & * & * & * & * & * & -\varepsilon I
\end{array}\right]<0,} \\
& {\left[\begin{array}{cc}
u_{\max }^{2} I & Y \\
* & Q
\end{array}\right] \geq 0,}
\end{aligned}
$$


where $\bar{L}=\varepsilon L$ and $\varepsilon>0$ is a positive scalar and $\Gamma=Q^{T} A+$ $\bar{\delta} Y^{T} B^{T}$.

At each future time instant, there exists the optimal control law to minimize the performance objective. Finally, the closed-loop system will be asymptotically stable.

Proof. In order to achieve the robust MPC performance objective under input constraint and packet dropouts, there exist three LMIs that need to be feasible. The first LMI is obtained by (16); by minimizing the upper bound of $\gamma$, we can get the second LMI; the third LMI is obtained by solving the input constraint (3). In the following, we give the proof.

Let $Q=\gamma P^{-1}>0$ and use Schur complement we can obtain inequality (28). Combining (4), (5), and (9) with (14), we have

$$
\begin{gathered}
E\left\{\hat { x } ^ { T } ( k + i | k ) \left[(A-L C)^{T} P(A-L C)\right.\right. \\
+\bar{\delta}(A-L C)^{T} P B K \\
+\bar{\delta} K^{T} B^{T} P(A-L C) \\
+\left(\bar{\delta}^{2}+\phi^{2}\right) K^{T} B^{T} P B K \\
\left.+C^{T} R_{1} C+\left(\bar{\delta}^{2}+\phi^{2}\right) K^{T} R_{2} K-P\right] \\
\times \widehat{x}(k+i \mid k)+\widehat{x}^{T}(k+i \mid k)\left[(A-L C)^{T} P L C\right. \\
\left.+\bar{\delta} K^{T} B^{T} P L C\right] \\
\times x(k+i \mid k)+x^{T}(k+i \mid k)\left[C^{T} L^{T} P(A-L C)\right. \\
\left.+\bar{\delta} C^{T} L^{T} P B K\right] \\
\left.\times \widehat{x}(k+i \mid k)+x^{T}(k+i \mid k) C^{T} L^{T} P L C x(k+i \mid k)\right\}
\end{gathered}
$$$$
<0 .
$$

Since $Q=\gamma P^{-1}$, inequality (30) is equivalent to

$$
\begin{aligned}
E\left\{\hat{x}^{T}(k+i \mid k)[\right. & (A-L C)^{T} Q^{-1}(A-L C) \\
& +\bar{\delta}(A-L C)^{T} Q^{-1} B K \\
& +\bar{\delta} K^{T} B^{T} Q^{-1}(A-L C) \\
& +\left(\bar{\delta}^{2}+\phi^{2}\right) K^{T} B^{T} Q^{-1} B K \\
& +\gamma^{-1} C^{T} R_{1} C \\
& \left.+\left(\bar{\delta}^{2}+\phi^{2}\right) \gamma^{-1} K^{T} R_{2} K-Q^{-1}\right]
\end{aligned}
$$

$$
\begin{aligned}
& \times \hat{x}(k+i \mid k)+\widehat{x}^{T}(k+i \mid k)\left[(A-L C)^{T} Q^{-1} L C\right. \\
& \left.+\bar{\delta} K^{T} B^{T} Q^{-1} L C\right] \\
& \times x(k+i \mid k)+x^{T}(k+i \mid k) \\
& \times\left[C^{T} L^{T} Q^{-1}(A-L C)+\bar{\delta} C^{T} L^{T} Q^{-1} B K\right] \widehat{x}(k+i \mid k) \\
& \left.+x^{T}(k+i \mid k) C^{T} L^{T} Q^{-1} L C x(k+i \mid k)\right\}
\end{aligned}
$$$$
<0,
$$

by pre-multiplying and post-multiplying (31) by $Q^{T}$ and $Q$, respectively. And (31) is satisfied if there exists a weighting positive-definite matrix $\Lambda$ such that

$$
\begin{aligned}
E\left\{Q ^ { T } \left\{\hat{x}^{T}(k+i \mid k)[\right.\right. & (A-L C)^{T} Q^{-1}(A-L C) \\
& +\bar{\delta}(A-L C)^{T} Q^{-1} B K \\
& +\bar{\delta} K^{T} B^{T} Q^{-1}(A-L C) \\
& +\left(\bar{\delta}^{2}+\phi^{2}\right) K^{T} B^{T} Q^{-1} B K \\
& +\gamma^{-1} C^{T} R_{1} C \\
& \left.+\left(\bar{\delta}^{2}+\phi^{2}\right) \gamma^{-1} K^{T} R_{2} K-Q^{-1}\right] \\
& \times \widehat{x}(k+i \mid k)+\widehat{x}^{T}(k+i \mid k) \\
\times & {\left[(A-L C)^{T} Q^{-1} L C+\bar{\delta} K^{T} B^{T} Q^{-1} L C\right] } \\
\times & x(k+i \mid k)+x^{T}(k+i \mid k) \\
\times & {\left[C^{T} L^{T} Q^{-1}(A-L C)+\bar{\delta} C^{T} L^{T} Q^{-1} B K\right] \widehat{x}(k+i \mid k) } \\
& \left.\left.+x^{T}(k+i k)\left[C^{T} L^{T} Q^{-1} L C-\Lambda\right] x(k+i \mid k)\right\} Q\right\}
\end{aligned}
$$

$<0$.

Inequality (32) is equivalent to

$$
\begin{aligned}
& E\left\{\theta^{T}(k+i \mid k)\left[\begin{array}{cc}
Q^{T} \Phi Q & Q^{T} \Psi^{T} Q^{-1} L C Q \\
Q^{T} C^{T} L^{T} Q^{-1} \Psi Q & Q^{T} C^{T} L^{T} Q^{-1} L C Q-\Lambda
\end{array}\right]\right. \\
& \quad \times \theta(k+i \mid k)\}<0,
\end{aligned}
$$


where

$$
\begin{aligned}
\Phi= & (A-L C)^{T} Q^{-1}(A-L C) \\
& +\bar{\delta}(A-L C)^{T} Q^{-1} B K \\
& +\bar{\delta} K^{T} B^{T} Q^{-1}(A-L C) \\
& +\left(\bar{\delta}^{2}+\phi^{2}\right) K^{T} B^{T} Q^{-1} B K
\end{aligned}
$$

$$
\begin{aligned}
& +\gamma^{-1} C^{T} R_{1} C+\left(\bar{\delta}^{2}+\phi^{2}\right) \gamma^{-1} K^{T} R_{2} K-Q^{-1}, \\
\Psi= & A-L C+\bar{\delta} B K, \\
\theta(k+i \mid k)= & {\left[\widehat{x}^{T}(k+i \mid k) x^{T}(k+i \mid k)\right]^{T} . }
\end{aligned}
$$

Inequality (33) is equivalent to

$$
\left[\begin{array}{cc}
Q^{T} \Phi Q & Q^{T}\left[(A-L C+\bar{\delta} B K) Q^{-1} L C\right] Q \\
Q^{T} C^{T} L^{T} Q^{-1}(A-L C+\bar{\delta} B K) Q & Q^{T} C^{T} L^{T} Q^{-1} L C Q-\Lambda
\end{array}\right]<0 .
$$

By using Schur complement repeatedly and Lemma 1, we can obtain the following inequality:

$$
\left[\begin{array}{cccccccc}
-Q & 0 & Q^{T} A+\bar{\delta} Y^{T} B^{T} & Y^{T} B^{T} & Y^{T} R_{2}^{1 / 2} & Q^{T} C^{T} R_{1}^{1 / 2} & Q^{T} C^{T} & 0 \\
* & -\Lambda & 0 & 0 & 0 & 0 & Q^{T} C^{T} & 0 \\
* & * & -Q & 0 & 0 & 0 & 0 & \bar{L} \\
* & * & * & \frac{-Q}{\phi^{2}} & 0 & 0 & 0 & 0 \\
* & * & * & * & \frac{-\gamma I}{\bar{\delta}^{2}+\phi^{2}} & 0 & 0 & 0 \\
* & * & * & * & * & -\gamma I & 0 & 0 \\
* & * & * & * & * & * & -\varepsilon I & 0 \\
* & * & * & * & * & * & * & -\varepsilon I
\end{array}\right]<0,
$$

where $Y=K Q$ and $\bar{L}=\varepsilon L$ and $\varepsilon>0$ is a positive scalar. Combining (3), (5), (9), (16), and (26), we have

$$
\begin{aligned}
\max _{i \geq 0}\|u(k+i \mid k)\|_{2}^{2} \\
\leq \max _{i \geq 0}\left\|u_{c}(k+i \mid k)\right\|_{2}^{2} \\
=\max _{i \geq 0}\left\|Y Q^{-1} \widehat{x}(k+i \mid k)\right\|_{2}^{2} \\
\leq \max _{i \geq 0}\left\|Y Q^{-1 / 2} Q^{-1 / 2} \widehat{x}(k+i \mid k)\right\|_{2}^{2} \\
=\lambda_{\max }\left(Q^{-1 / 2} Y^{T} Y Q^{-1 / 2}\right) \leq u_{\max }^{2} .
\end{aligned}
$$

By using Schur complement, we can obtain

$$
\left[\begin{array}{cc}
u_{\max }^{2} I & * \\
Y^{T} & Q
\end{array}\right] \geq 0
$$

\section{Dynamic Output Controller}

Theorem 4. Assume that the uncertainty $\Omega$ is a prescribed polytope and the probabilities $\bar{\alpha}$ and $\bar{\beta}$ take values from 0 to 1 .
The control input (5) under a series of constrained inputs (3) that minimizes $J_{\infty}(k)$ is obtained by

$$
\begin{aligned}
A_{c} & =G_{1} W_{2}^{-1}, \\
B_{c} & =\widetilde{B} C^{-1}, \\
C_{c} & =G_{2} W_{2}^{-1},
\end{aligned}
$$

where $W_{2}>0$ is symmetric and the upper bound $\gamma$ of $J_{\infty}(k)$ can be solved by the following minimization problem:

$$
\min _{u(k \mid k), Q, Y} \gamma
$$

subject to

$$
\begin{aligned}
& {\left[\begin{array}{cc}
1 & \theta^{T}(k \mid k) \\
* & W
\end{array}\right]>0,} \\
& {\left[\begin{array}{ll}
\Upsilon_{1} & \Upsilon_{2} \\
* & \Upsilon_{3}
\end{array}\right]>0 \text {, }}
\end{aligned}
$$

$$
\left[\begin{array}{cc}
W & \widetilde{G}_{2}^{T} \\
* & u_{\max }^{2} I
\end{array}\right] \geq 0,
$$

where 


$$
\begin{gathered}
\Upsilon_{1}=\left[\begin{array}{cccccccc}
-W_{1} & 0 & W_{1}^{T} A^{T} & 0 & 0 & 0 & W_{1}^{T} C^{T} R_{1}^{1 / 2} & 0 \\
* & -W_{2} & \bar{\beta} G_{2}^{T} B^{T} & G_{1}^{T} & q G_{2}^{T} B^{T} & 0 & 0 & W_{2}^{T} C_{c}^{T} R_{2}^{1 / 2} \\
* & * & -W_{1} & 0 & 0 & 0 & 0 & 0 \\
* & * & * & -W_{2} & 0 & 0 & 0 & 0 \\
* & * & * & * & -W_{1} & 0 & 0 & 0 \\
* & * & * & * & * & -W_{2} & 0 & 0 \\
* & * & * & * & * & * & -\gamma I & 0 \\
* & * & * & * & * & * & * & \frac{-\gamma I}{S}
\end{array}\right], \\
\Upsilon_{2}=\left[\begin{array}{cccccccc}
C W_{1} & 0 & 0 & 0 & 0 & 0 & 0 & 0 \\
0 & 0 & 0 & \bar{\alpha} \widetilde{B}^{T} & 0 & l \widetilde{B}^{T} & 0 & 0
\end{array}\right]^{T},
\end{gathered}
$$

$A_{c}=G_{1} W_{2}^{-1}, B_{c}=\widetilde{B} C^{-1}, C_{c}=G_{2} W_{2}^{-1}, \bar{\varepsilon}>0$ is a positive scalar, and $\widetilde{G}_{2}=\left[\begin{array}{ll}0 & G_{2}\end{array}\right]$.

Proof. Let $W=\gamma P^{-1}>0$ and, using Schur complement, we can obtain inequality (41). Combining (10) and (7) with (18), we have

$$
\begin{aligned}
& E\left\{\theta ^ { T } ( k + i | k ) \left[\bar{A}^{T}(k) P \bar{A}(k)+\widehat{A}^{T}(k) P \widehat{A}(k)\right.\right. \\
& <0,
\end{aligned}
$$

by premultiplying and postmultiplying (46) by $W^{T}$ and $W$, respectively. By further transformation, we can achieve

$$
\begin{aligned}
-W & +\bar{W}+W^{T} \bar{B}^{T} W^{-1} \bar{B} W+W^{T} \widetilde{C}^{T}\left(\gamma R_{1}^{-1}\right) \widetilde{C} W \\
& +s W^{T} \widetilde{C}_{c}^{T}\left(\gamma R_{2}^{-1}\right) \widetilde{C}_{c} W<0,
\end{aligned}
$$

where $\bar{B}=\left[\begin{array}{cc}0 & m B C_{c} \\ l B_{c} C & 0\end{array}\right], \widetilde{C}_{c}=\left[\begin{array}{ll}0 & C_{c}\end{array}\right], \bar{W}=$ $W^{T} \bar{A}^{T}(k) W^{-1} \bar{A}(k)$, and $s=\bar{\beta}^{2}+m^{2}$. By using Schur complement repeatedly and Lemma 1 , we can obtain the following inequality:

$$
\left[\begin{array}{cccccccccc}
-W_{1} & 0 & W_{1}^{T} A^{T} & 0 & 0 & 0 & \widehat{W} & 0 & W_{1}^{T} C^{T} & 0 \\
* & -W_{2} & \bar{\beta} G_{2}^{T} B^{T} & G_{1}^{T} & \check{G} & 0 & 0 & \check{W} & 0 & 0 \\
* & * & -W_{1} & 0 & 0 & 0 & 0 & 0 & 0 & 0 \\
* & * & * & -W_{2} & 0 & 0 & 0 & 0 & 0 & \bar{\alpha} \widetilde{B} \\
* & * & * & * & -W_{1} & 0 & 0 & 0 & 0 & 0 \\
* & * & * & * & * & -W_{2} & 0 & 0 & 0 & l \widetilde{B} \\
* & * & * & * & * & * & -\gamma I & 0 & 0 & 0 \\
* & * & * & * & * & * & * & -\gamma I & 0 & 0 \\
* & * & * & * & * & * & * & * & -\bar{\varepsilon} I & 0 \\
* & * & * & * & * & * & * & * & * & -\bar{\varepsilon} I
\end{array}\right]>0,
$$

where $\widehat{W}=W_{1}^{T} C^{T} R_{1}^{1 / 2}, \check{G}=q G_{2}^{T} B^{T}, \check{W}=W_{2}^{T} C_{c}^{T} R_{2}^{1 / 2}$, and $\bar{\varepsilon}>0$ is a positive scalar.

\section{Simulation Results}

Consider the following uncertain polytope system:

$$
\begin{aligned}
& A_{1}=\left[\begin{array}{cc}
0.01942 & 0 \\
-0.5100 & 0.02000
\end{array}\right], \\
& A_{2}=\left[\begin{array}{cc}
0.01942 & 0 \\
-0.5100 & 0.01000
\end{array}\right],
\end{aligned}
$$

$$
\begin{aligned}
& B_{1}=B_{2}=B=\left[\begin{array}{ll}
1 & -0.0787
\end{array}\right]^{T}, \\
& C_{1}=C_{2}=C=\left[\begin{array}{ll}
1 & -0.4
\end{array}\right], \\
& D_{1}=D_{2}=D=0,
\end{aligned}
$$

where the polytope formed by the two local discrete models is as follows:

$$
[A(k) B(k)] \in \Omega=\operatorname{Co}\left\{\left[\begin{array}{ll}
A_{1} & B
\end{array}\right],\left[\begin{array}{ll}
A_{2} & B
\end{array}\right]\right\} .
$$




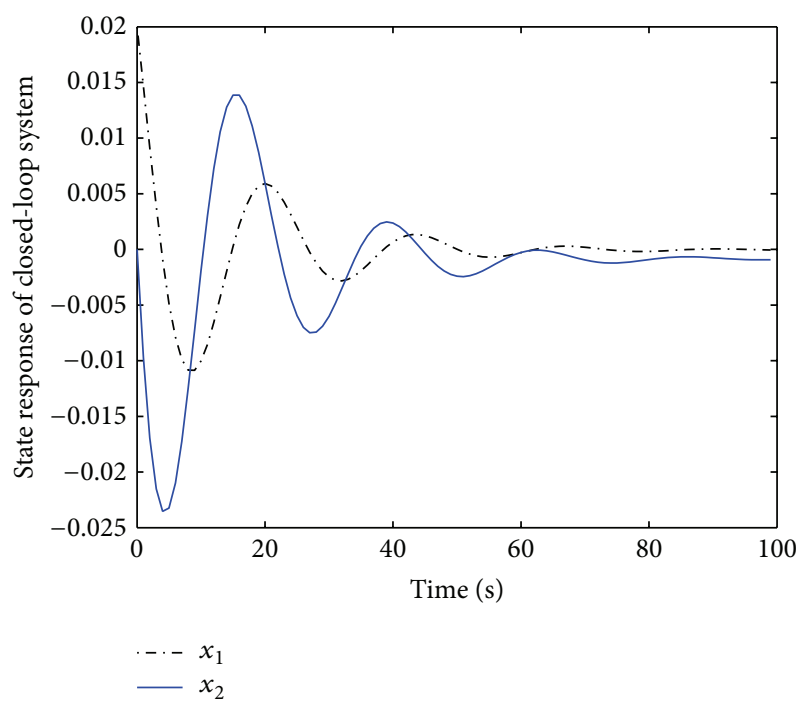

Figure 1: The state response of closed-loop system.

In the simulation, let $\left[\begin{array}{ll}A(k) & B(k)\end{array}\right]=\left[\begin{array}{ll}A_{1} & B\end{array}\right]$. The input constraint is as follows:

$$
|u(k)| \leq 0.25, \quad k \geq 0 .
$$

In Theorem 2 , the $\bar{L}_{D i}$ decay rate $\rho$ and weighting matrix $\widetilde{L}$ are given as

$$
\rho^{2}=0.8, \quad \widetilde{L}=\left[\begin{array}{cc}
0.1 & 0 \\
0 & 0.1
\end{array}\right]
$$

Then, the infinite horizon robust performance objective $J_{\infty}(k)$ has the following weighting matrices: $R_{1}=0.01, R_{2}=$ 0.00001 .

Another weighting matrix $\Lambda$ is given as

$$
\Lambda=\left[\begin{array}{cc}
0.01 & 0 \\
0 & 0.1
\end{array}\right]
$$

where the initial states of the real system (1) and the state observer are $x_{0}=\left[\begin{array}{ll}0.02 & 0\end{array}\right]^{T}$ and $\widehat{x}_{0}=\left[\begin{array}{ll}0.02 & -0.01\end{array}\right]^{T}$, respectively.

The state observer gain $L$ and MPC controller gain $K$ are obtained as $L=\left[\begin{array}{ll}0.0167 & -0.4458\end{array}\right]^{T}$ and $K=\left[\begin{array}{l}-0.7147 \\ 0\end{array}\right.$ 0.4889 ], respectively.

In Figure 1, the two curves are the state of the original closed-loop system. The solid line represents the real state $x_{i}(k)(i=1,2)$ of the original closed-loop system and the dashed line denotes its estimated state $\widehat{x}_{i}(k)(i=1,2)$, respectively, in Figures 2 and 3. In Figure 4, the curve denotes the control input $u(k)$ under the robust performance objective with the constrained input. The curve shows the output of closed-loop system, which converges to zero when $k \rightarrow \infty$ in Figure 5 .

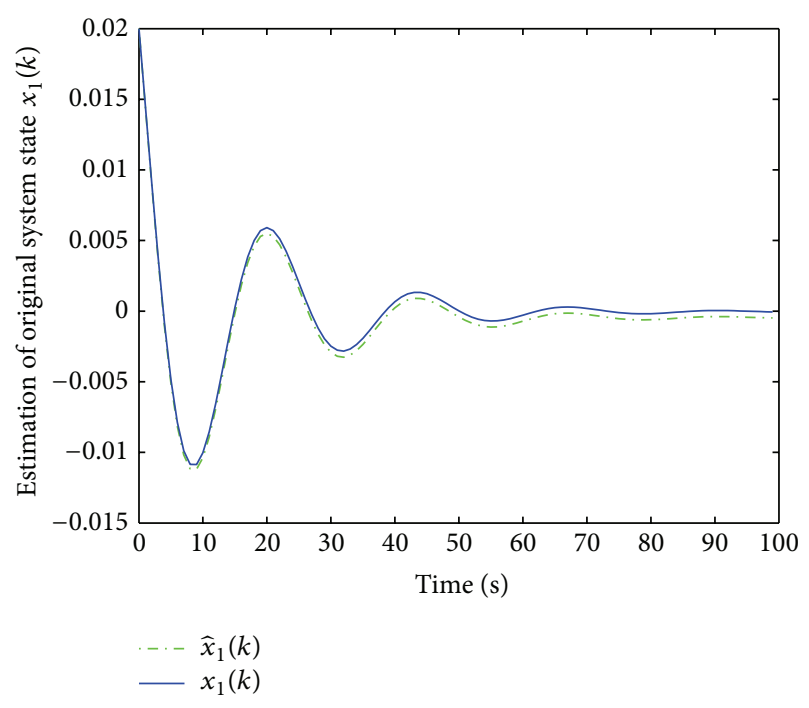

FIGURE 2: Estimation of original system state $x_{1}(k)$.

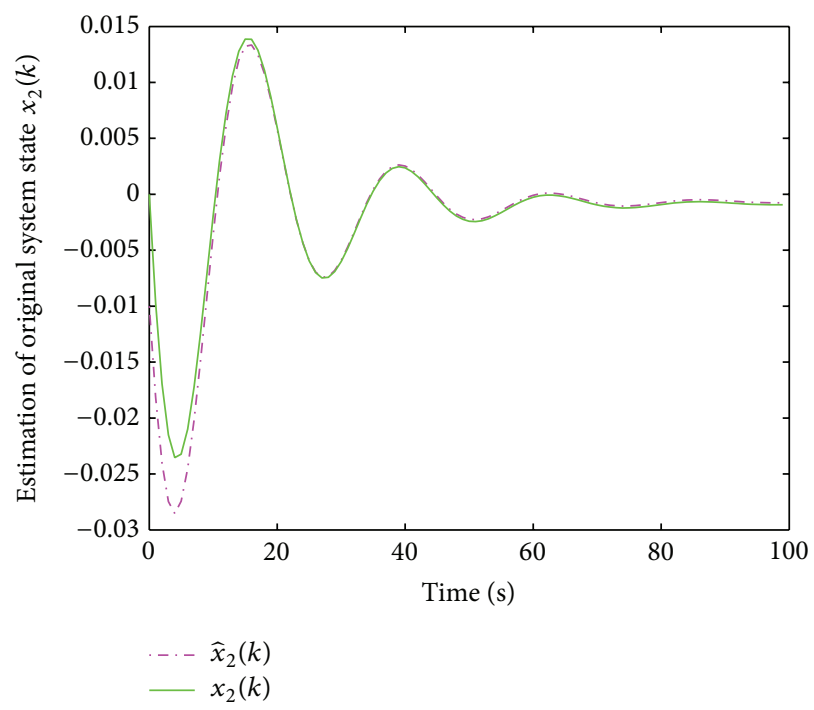

FIGURE 3: Estimation of original system state $x_{2}(k)$.

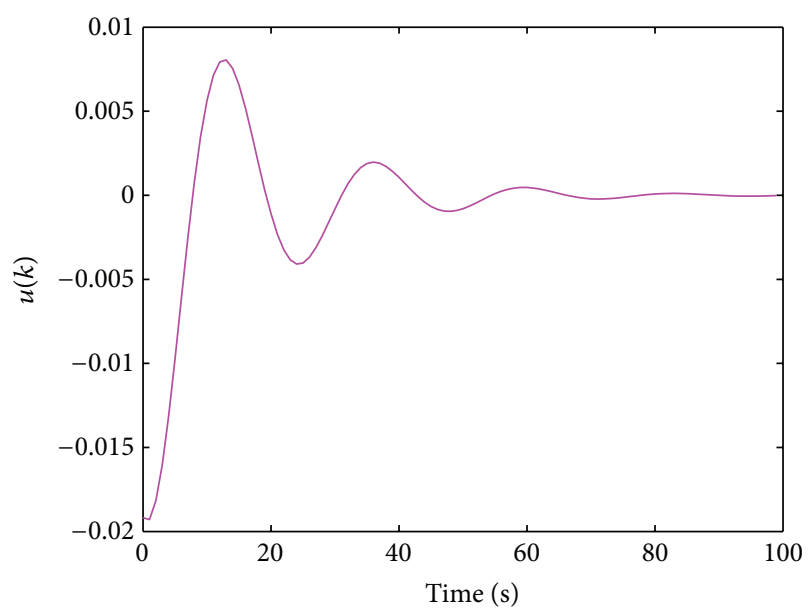

FIgURE 4: The control input signal $u(k)$. 


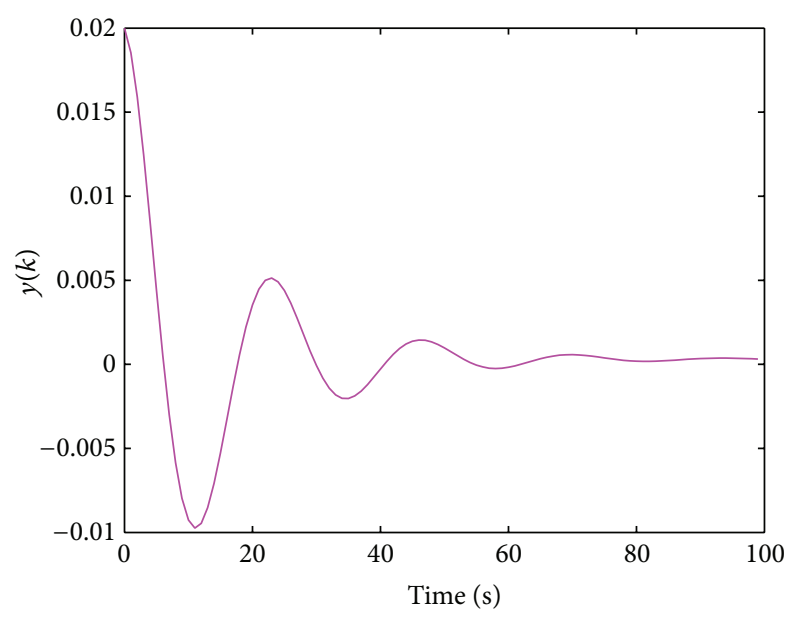

Figure 5: The output of closed-loop system.

Consider the following uncertain polytope system:

$$
\begin{gathered}
A_{1}=\left[\begin{array}{cc}
0.04942 & -0.01 \\
0 & 0.03000
\end{array}\right], \\
A_{2}=\left[\begin{array}{cc}
0.04942 & -0.01 \\
0 & 0.01000
\end{array}\right], \\
B_{1}=B_{2}=B=\left[\begin{array}{ll}
-0.43 & 0
\end{array}\right]^{T}, \\
C_{1}=C_{2}=C=\left[\begin{array}{ll}
1 & 0.4
\end{array}\right], \\
D_{1}=D_{2}=D=0,
\end{gathered}
$$

where the polytope formed by the two local discrete models is as follows:

$$
[A(k) B(k)] \in \Omega=\operatorname{Co}\left\{\left[\begin{array}{ll}
A_{1} & B
\end{array}\right],\left[\begin{array}{ll}
A_{2} & B
\end{array}\right]\right\} .
$$

In the simulation, let $[A(k) B(k)]=\left[\begin{array}{ll}A_{1} & B\end{array}\right]$. The input constraint is as follows:

$$
|u(k)| \leq 0.2, \quad k \geq 0 .
$$

Then, the infinite horizon robust performance objective $J_{\infty}(k)$ has the following weighting matrices:

$$
R_{1}=1, \quad R_{2}=0.8,
$$

where the initial states of the real system (1) and the dynamic output controller are $x_{0}=\left[\begin{array}{ll}0 & -0.00001\end{array}\right]^{T}$ and $\widehat{x}_{c}=\left[\begin{array}{ll}0.35 & -\end{array}\right.$ $0.09]^{T}$, respectively.

The dynamic output controller gains, $A_{c}, B_{c}$, and $C_{c}$, are obtained as

$$
\begin{aligned}
& A_{c}=\left[\begin{array}{cc}
-0.4597 & -0.0821 \\
0.1933 & -0.2124
\end{array}\right], \\
& B_{c}=\left[\begin{array}{l}
0.3845 \\
0.1024
\end{array}\right], \\
& C_{c}=\left[\begin{array}{ll}
-0.0187 & 0.0850
\end{array}\right] .
\end{aligned}
$$

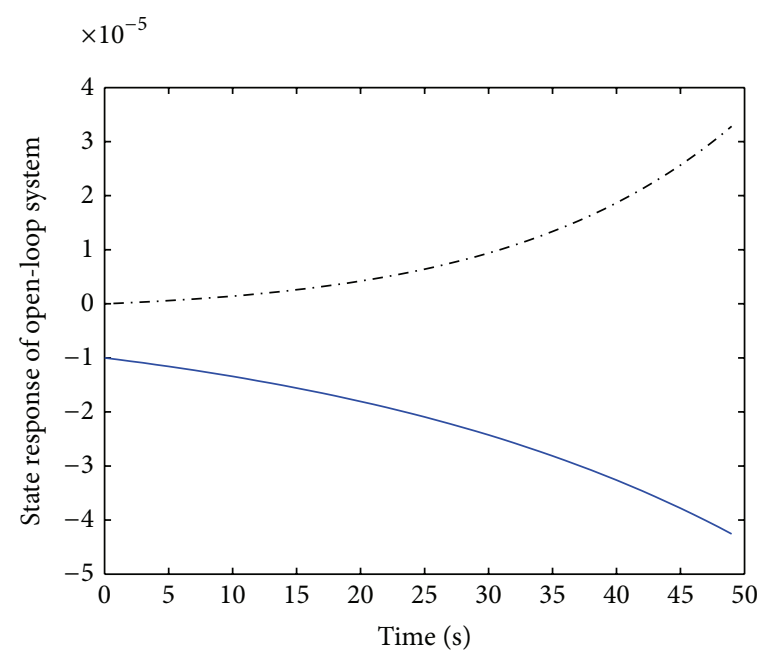

$\cdot-\cdot-x_{1}$

FIGURE 6: The state response of open-loop system.

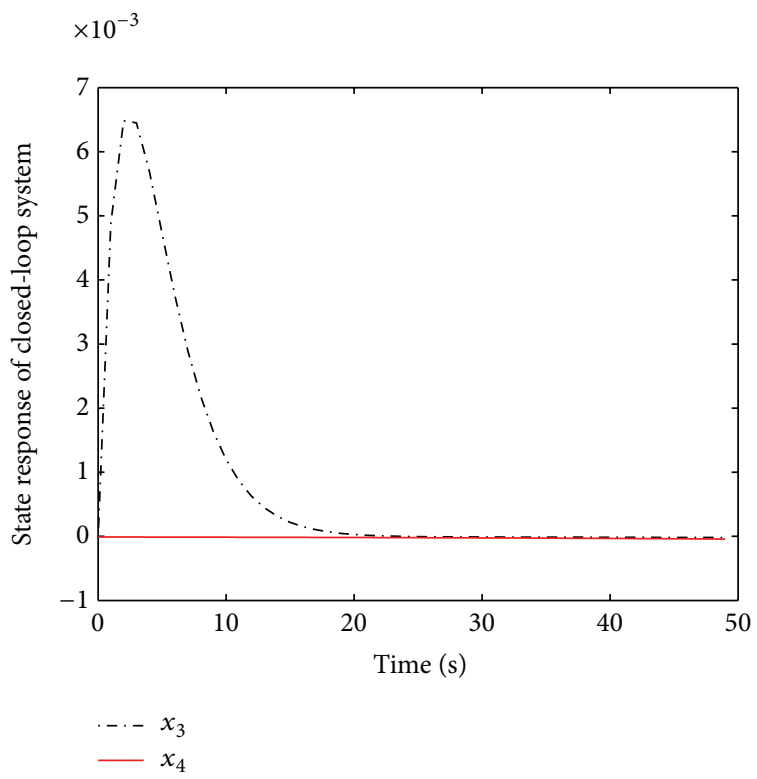

Figure 7: The state response of closed-loop system.

In Figure 6, the two curves are the state of the original open-loop system. In Figure 7, the two curves are the state of the original closed-loop system. In Figure 8, the two curves are the state of the dynamic output controller. The curve denotes the control input $u(k)$ under the robust performance objective with the constrained input in Figure 9. The curve represents the control output $u_{c}(k)$ of dynamic output controller in Figure 10. The curve shows the output of closed-loop system, which converges to zero when $k \rightarrow \infty$ in Figure 11 .

\section{Conclusion}

In this paper, we have designed an output-feedback MPC to solve the problem of the robust MPC with input constraints and successive packet dropouts. The method makes use of 


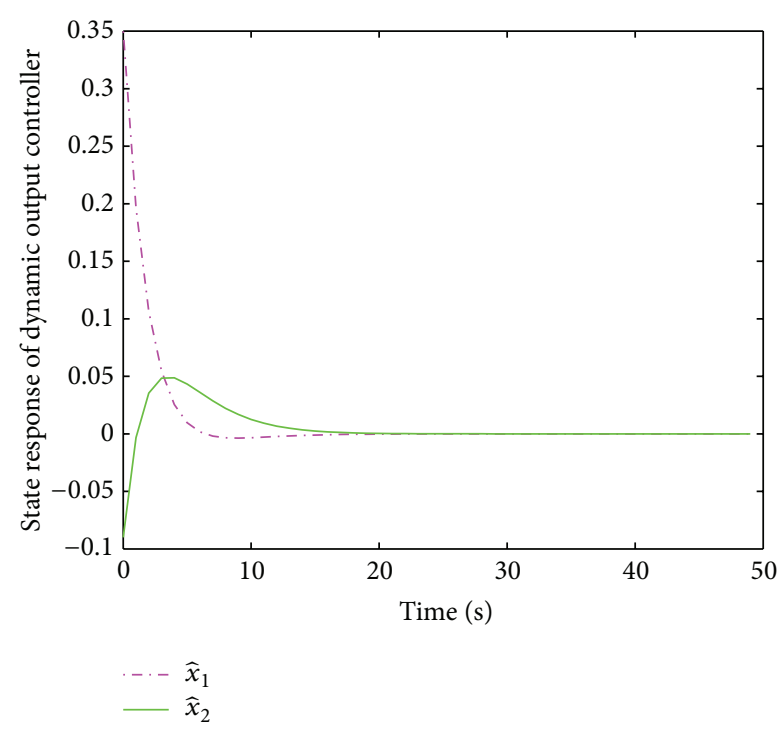

Figure 8: The state response of dynamic output controller.

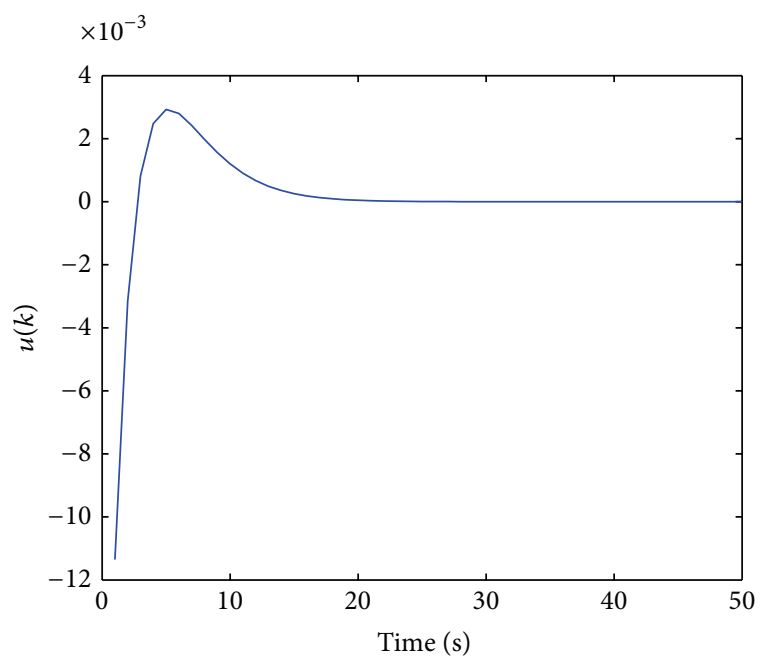

FIgURE 9: The control input signal $u(k)$.

infinite horizon min-max algorithm with LMI constraints. First, we have constructed a state observer. Then, the optimization problem can be solved by dealing with some LMI constraints. We can obtain the control input sequence by dealing with the infinite horizon robust MPC and input constraint based on the estimated state of observer. From the simulation, the design method of robust MPC with input constraint has been verified feasiblely. As a future work, we will develop the output-feedback MPC algorithm combined with nonlinear MPC and its optimization.

\section{Conflict of Interests}

The authors declare that there is no conflict of interests regarding the publication of this paper.

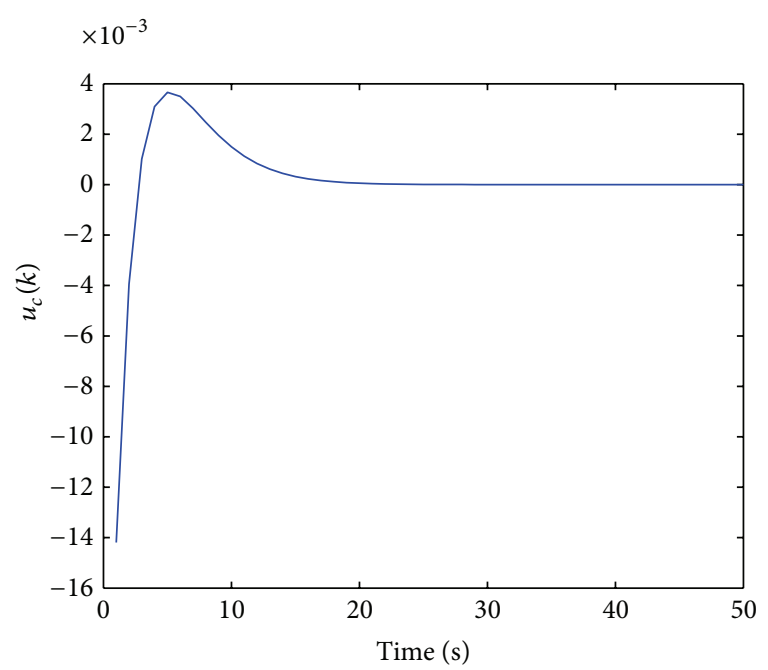

Figure 10: The control output signal $u_{c}(k)$ of dynamic output controller.

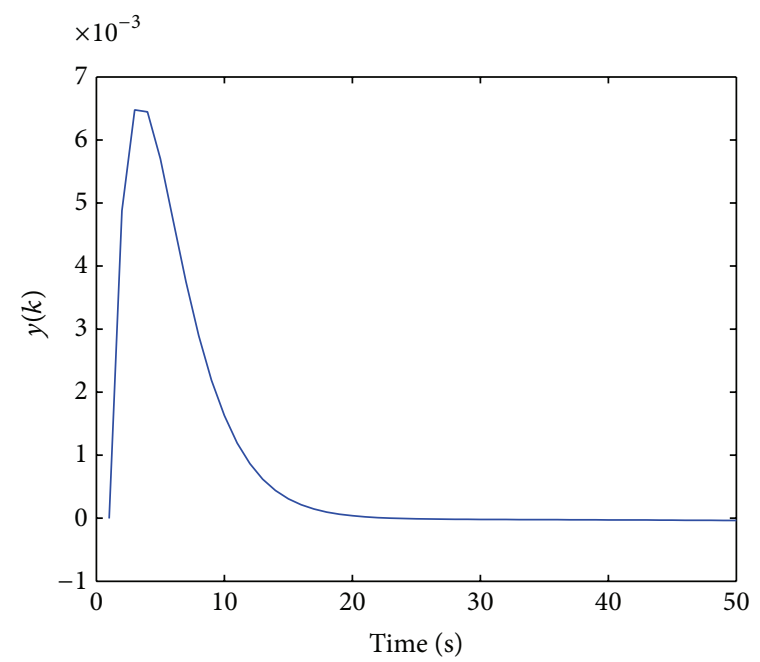

FIGURE 11: The output of closed-loop system.

\section{References}

[1] E. F. Camacho and C. B. Alba, Model Predictive Control, Springer, 2013.

[2] P. J. Campo and M. Morari, "Robust model predictive control," in Proceedings of the American Control Conference, pp. 10211026, IEEE, 1987.

[3] R. Yang, G. Liu, P. Shi, C. Thomas, and M. V. Basin, "Predictive output feedback control for networked control systems," IEEE Transactions on Industrial Electronics, vol. 61, no. 1, pp. 512-520, 2014.

[4] D. Q. Mayne, J. B. Rawlings, C. V. Rao, and P. O. M. Scokaert, "Constrained model predictive control: stability and optimality," Automatica, vol. 36, no. 6, pp. 789-814, 2000.

[5] Y. Xia, H. Yang, P. Shi, and M. Fu, "Constrained infinite-horizon model predictive control for fuzzy-discrete-time systems," IEEE Transactions on Fuzzy Systems, vol. 18, no. 2, pp. 429-436, 2010. 
[6] A. Casavola, M. Giannelli, and E. Mosca, "Min-max predictive control strategies for input-saturated polytopic uncertain systems," Automatica, vol. 36, no. 1, pp. 125-133, 2000.

[7] J. H. Lee and Z. Yu, "Worst-case formulations of model predictive control for systems with bounded parameters," Automatica, vol. 33, no. 5, pp. 763-781, 1997.

[8] S. Yin, H. Luo, and S. X. Ding, "Real-time implementation of fault-tolerant control systems with performance optimization," IEEE Transactions on Industrial Electronics, vol. 61, no. 5, pp. 2402-2411, 2013.

[9] S. Rakovic, Robust control of constrained discrete time systems: characterization and implementation [Ph.D. thesis], Imperial College London, University of London, 2005.

[10] H. R. Karimi, "Robust $H_{\infty}$ filter design for uncertain linear systems over network with network-induced delays and output quantization," Modeling, Identification and Control, vol. 30, no. 1, pp. 27-37, 2009.

[11] S. Yin, S. X. Ding, A. Haghani, H. Hao, and P. Zhang, "A comparison study of basic data-driven fault diagnosis and process monitoring methods on the benchmark Tennessee Eastman process," Journal of Process Control, vol. 22, no. 9, pp. 1567-1581, 2012.

[12] S. Yin, S. X. Ding, A. H. A. Sari, and H. Hao, "Data-driven monitoring for stochastic systems and its application on batch process," International Journal of Systems Science, vol. 44, no. 7, pp. 1366-1376, 2013.

[13] H. Li, X. Jing, H.-K. Lam, and P. Shi, "Fuzzy sampled-data control for uncertain vehicle suspension systems," IEEE Transactions on Cybernetics, vol. 44, no. 7, pp. 1111-1126, 2014.

[14] H. Li, H. Liu, H. Gao, and P. Shi, "Reliable fuzzy control for active suspension systems with actuator delay and fault," IEEE Transactions on Fuzzy Systems, vol. 20, no. 2, pp. 342-357, 2012.

[15] H. Li, X. Jing, and H. R. Karimi, "Output-feedback-based $\mathrm{H}_{\infty}$ control for vehicle suspension systems with control delay," IEEE Transactions on Industrial Electronics, vol. 61, no. 1, pp. 436-446, 2014.

[16] H. Li, J. Yu, C. Hilton, and H. Liu, "Adaptive sliding-mode control for nonlinear active suspension vehicle systems using $\mathrm{T}$ S fuzzy approach," IEEE Transactions on Industrial Electronics, vol. 60 , no. 8, pp. 3328-3338, 2013.

[17] H. Li, H. Gao, P. Shi, and X. Zhao, "Fault-tolerant control of Markovian jump stochastic systems via the augmented sliding mode observer approach," Automatica, 2014.

[18] F. A. Cuzzola, J. C. Geromel, and M. Morari, "An improved approach for constrained robust model predictive control," Automatica, vol. 38, no. 8, pp. 1183-1189, 2002.

[19] S. J. Qin and T. A. Badgwell, "A survey of industrial model predictive control technology," Control Engineering Practice, vol. 11, no. 7, pp. 733-764, 2003.

[20] Y. J. Wang and J. B. Rawlings, "A new robust model predictive control method I: theory and computation," Journal of Process Control, vol. 14, no. 3, pp. 231-247, 2004.

[21] P. J. Campo and M. Morari, " $\infty$-norm formulation of model predictive control problems," in Proceedings of the IEEE American Contro Conference, pp. 339-343, Seattle, Wash, USA, June 1986.

[22] M. V. Kothare, V. Balakrishnan, and M. Morari, "Robust constrained model predictive control using linear matrix inequalities," Automatica, vol. 32, no. 10, pp. 1361-1379, 1996.

[23] Z. Wan and M. V. Kothare, "Robust output feedback model predictive control using off-line linear matrix inequalities," Journal of Process Control, vol. 12, no. 7, pp. 763-774, 2002.
[24] H. Gao and T. Chen, "Network-based $H_{\infty}$ output tracking control," IEEE Transactions on Automatic Control, vol. 53, no. 3, pp. 655-667, 2008.

[25] Y. Zhao, H. Gao, J. Lam, and B. Du, "Stability and stabilization of delayed T-S fuzzy systems: a delay partitioning approach," IEEE Transactions on Fuzzy Systems, vol. 17, no. 4, pp. 750-762, 2009.

[26] H. R. Karimi, N. A. Duffie, and S. Dashkovskiy, "Local capacity $H_{\infty}$ control for production networks of autonomous work systems with time-varying delays," IEEE Transactions on Automation Science and Engineering, vol. 7, no. 4, pp. 849-857, 2010.

[27] H. R. Karimi, "Delay-range-dependent linear matrix inequality approach to quantized $H_{\infty}$ control of linear systems with network-induced delays and norm-bounded uncertainties," Proceedings of the Institution of Mechanical Engineers I: Journal of Systems and Control Engineering, vol. 224, no. 6, pp. 689-700, 2010.

[28] R. Yang, P. Shi, G. P. Liu, and H. Gao, "Network-based feedback control for systems with mixed delays based on quantization and dropout compensation," Automatica, vol. 47, no. 12, pp. 2805-2809, 2011.

[29] H. Gao and T. Chen, "Stabilization of nonlinear systems under variable sampling: a fuzzy control approach," IEEE Transactions on Fuzzy Systems, vol. 15, no. 5, pp. 972-983, 2007.

[30] H. Gao, Y. Zhao, J. Lam, and K. Chen, " $\mathrm{H}_{b m \infty}$ fuzzy filtering of nonlinear systems with intermittent measurements," IEEE Transactions on Fuzzy Systems, vol. 17, no. 2, pp. 291-300, 2009.

[31] T. Chai, L. Zhao, J. Qiu, F. Liu, and J. Fan, "Integrated networkbased model predictive control for setpoints compensation in industrial processes," IEEE Transactions on Industrial Informatics, vol. 9, no. 1, pp. 417-426, 2013.

[32] J. Zhang, Y. Xia, and P. Shi, "Design and stability analysis of networked predictive control systems," IEEE Transactions on Control Systems Technology, vol. 21, no. 4, pp. 1495-1501, 2013.

[33] S. Sun, "Linear optimal state and input estimators for networked control systems with multiple packet dropouts," International Journal of Innovative Computing, Information and Control, vol. 8, no. 10, pp. 7289-7305, 2012.

[34] D. Q. Mayne, S. V. Raković, R. Findeisen, and F. Allgöwer, "Robust output feedback model predictive control of constrained linear systems: time varying case," Automatica, vol. 45, no. 9, pp. 2082-2087, 2009.

[35] R. Moitie, M. Quincampoix, and V. M. Veliov, "Optimal control of discrete-time uncertain systems with imperfect measurement," Institute of Electrical and Electronics Engineers: Transactions on Automatic Control, vol. 47, no. 11, pp. 1909-1914, 2002. 


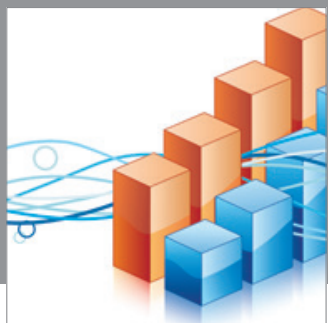

Advances in

Operations Research

mansans

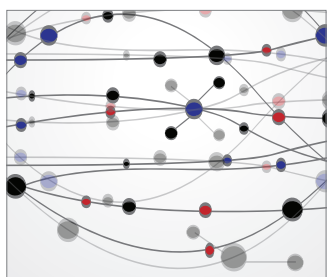

The Scientific World Journal
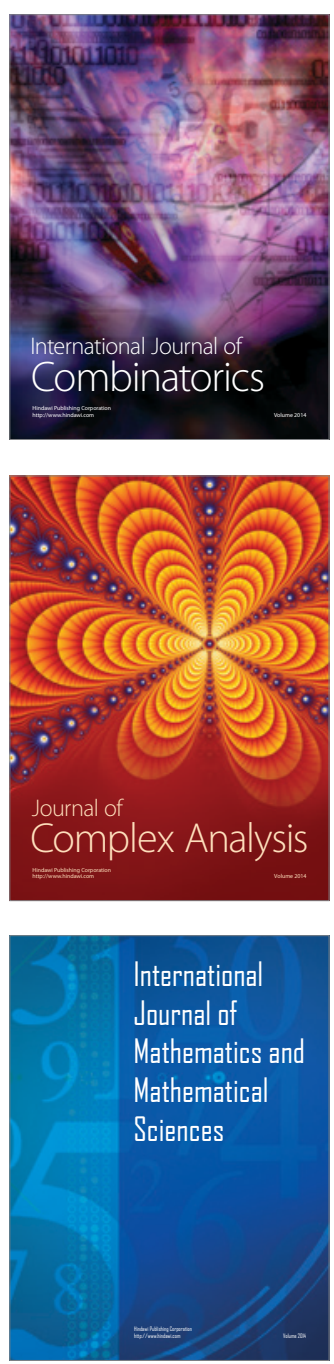
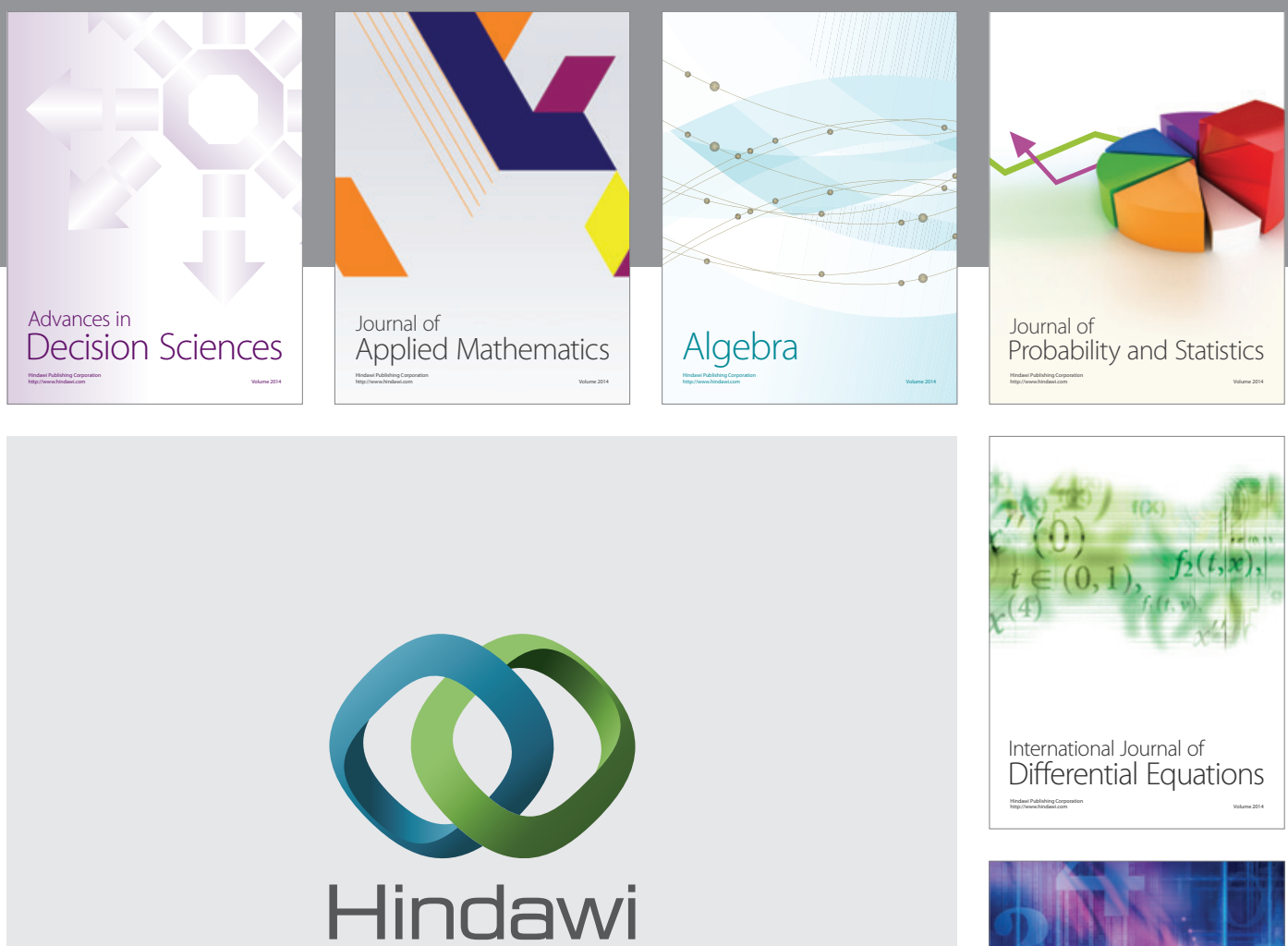

Submit your manuscripts at http://www.hindawi.com
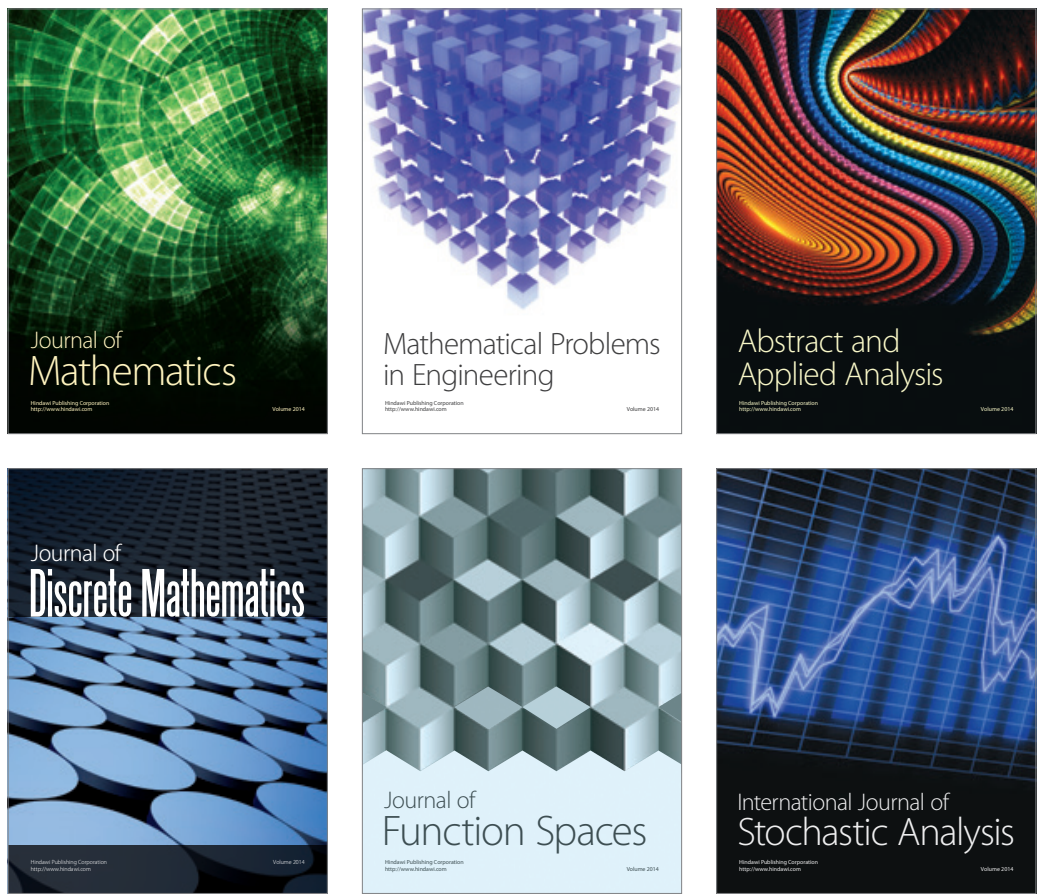

Journal of

Function Spaces

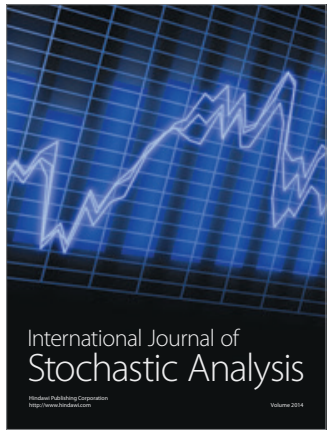

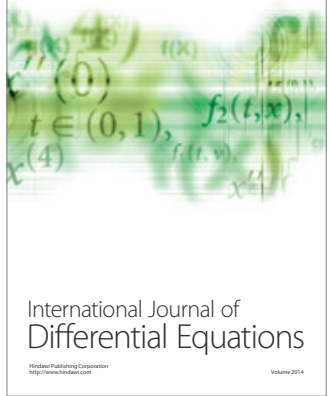
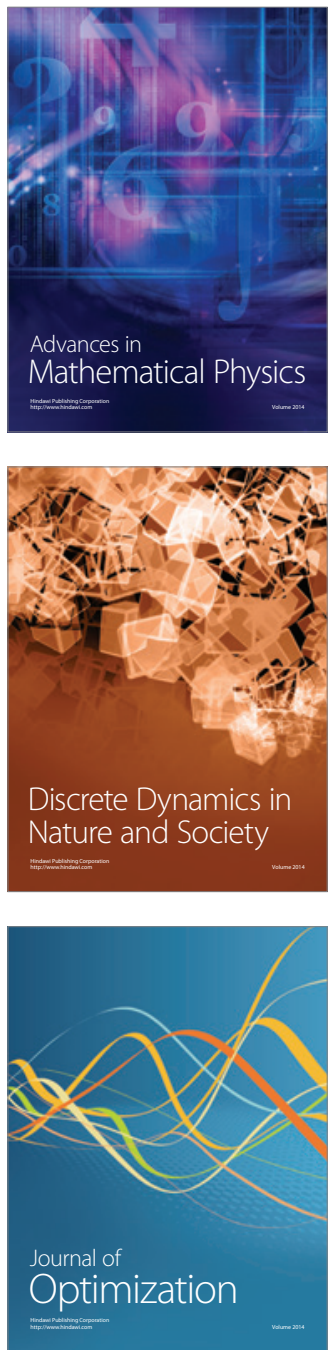